\title{
Preparation and characterization of polysaccharide-based nanoparticles with anticoagulant activity
}

This article was published in the following Dove Press journal:

International Journal of Nanomedicine

16 June 2012

Number of times this article has been viewed

\author{
Luiz Cláudio da Silva' \\ Thiago Garcia' \\ Michela Mori ${ }^{2}$ \\ Giuseppina Sandri² \\ Maria Cristina Bonferoni ${ }^{2}$ \\ Priscila $V$ Finotelli' \\ Leonardo P Cinelli' \\ Carla Caramella ${ }^{2}$ \\ Lúcio M Cabral' \\ 'Drug Department, Rio de Janeiro \\ Federal University, Rio de Janeiro, \\ Brazil; ${ }^{2}$ Department of Drug Sciences, \\ University of Pavia, Pavia, Italy
}

Correspondence: Luiz Cláudio da Silva Departmento de Medicamentos, Universidade Federal do Rio de Janeiro, Avenida Carlos Chagas Filho 373, CEP 21.94I-902, Rio de Janeiro, RJ, Brazil Tel +55 2I 25626605

Fax +552125626605

Email lulaufrj@hotmail.com
Abstract: The aim of this study was to produce and characterize nanoparticles (NPs), combining chondroitin sulfate (CS) and fucoidan (FC) with chitosan for therapeutic purposes. These NPs were characterized by dynamic light scattering, zeta potential determination, and transmission electronic microscopy. The anticoagulant activity was determined for FC NPs and compared with FC solution at the same concentration. FC NPs showed regular shapes and better anticoagulant activity than free polysaccharide solution. FC solution did not affect coagulation compared to FC NPs, which increased up to two-fold, even at a lower concentration. Cytotoxicity and permeability tests were conducted using Caco-2 cell monolayer, exhibiting no toxic effect in this cell line and higher permeability for NP2 samples than FC solution at the same concentration.

Keywords: nanoparticles, chitosan, chondroitin sulfate, fucoidan, aPTT, Caco-2

\section{Introduction}

Sulfated polysaccharides are found in many animals (vertebrates and invertebrates) and plant tissues with different polyanionic structures. This feature is due to the presence of sulfate esters and/or hexuronic acid. These macromolecules are largely studied for their interesting biological profile, including anticoagulant activity. ${ }^{1-3}$ Sulfated polysaccharides occur naturally as acidic polysaccharides. Among them, chondroitin sulfate (CS) is an abundant glycosaminoglycan found in bone, cartilage, and connective mammalian tissue. It is composed of $\mathrm{N}$-acetylgalactosamine and glucuronic acid residues linked by 1-3 generally sulfated on $N$-acetylgalactosamine at 4- or 6-position. Chondroitin sulfate is able to produce ionic complexes, but only with positively charged molecules, due to its acidic mucopolysaccharide structure. ${ }^{4-7}$ This polysaccharide has recognized anti-inflammatory and antitumor activities and can be used in combination with other polysaccharides in different hydrogel drug delivery systems. ${ }^{8,9}$ Another interesting sulfated polysaccharide is the sulfated fucan, which displays anticoagulant and antithrombotic activities. ${ }^{1,10,11}$ Also known as fucoidan (FC), ${ }^{12}$ this substance is found in brown algae (Fucus vesiculosus) and displays an ability to increase the inhibition of thrombin by antithrombin or heparin cofactor II. ${ }^{1,2}$ Biopolymers such as CT are considered natural complexing agents, with the ability to form physical gels or coacervates with sulfated polysaccharides. Chitosan is a linear polysaccharide, composed of units of D-glucosamine and N-acetyl-D-glucosamine, which in acidic $\mathrm{pH}$ is in a cationic form..$^{5,13-19}$ The properties of CT have been studied extensively for its mucoadhesion to the gastrointestinal mucosal epithelium and anti-inflammatory activity. ${ }^{20,21} \mathrm{CT}$ produces insoluble ionic complexes with water-soluble polyanionic species in neutral or 
alkaline medium. Some negative polyelectrolytes, such as alginate, carboxymethylcellulose, xanthan gum, heparin, and $\mathrm{CS}$, are able to complex with $\mathrm{CT} .^{16,22} \mathrm{CS}$ and FC had never been used for the preparation of nanostructured systems with potential antithrombotic and anticoagulant activities. Nanostructured systems play an important role in future therapies because they are widely described in the literature as systems that cross the gastrointestinal epithelial absorption barrier. Moreover, NPs are able to control and direct the release of bioactive substances. ${ }^{23,24}$ In nanomedicine, a drug (synthetic or a biopolymer) can be dissolved, incorporated, encapsulated and/or adsorbed or attached to NPs in order to (1) improve its pharmacokinetic profile, ${ }^{24-27}$ (2) raise the effectiveness of treatment; ${ }^{28}$ (3) reduce the adverse effects of preferential accumulation in specific sites, causing low concentrations in healthy tissues $;{ }^{29}$ and (4) increase the chemical and conformational stability of a variety of therapeutic agents, such as small molecules, peptides, and oligonucleotides.

Particle size and zeta potential (ZP) are fundamental for colloidal system stabilization. Furthermore, particle absorption due to internalization/endocytosis is also driven by size (dimension) and surface chemistry (ZP), which allow an interaction with cells. In particular, surface chemistry critically affects how NPs interact with each other and the environment and, even more, with cells. Simply classifying the internalization of NPs in terms of their surface charge, neutral and anionic nanoparticles are internalized considerably less efficiently than cationic ones. Therefore, particle interaction with cell membranes has a drastic effect on the uptake process. ${ }^{30}$

In fact, the attractive interactions lead to a clustering that effectively lowers the free energy threshold for wrapping and, therefore, shifts the lower cutoff radius. When these are repulsed, the minimum radius for endocytosis increases, therefore hindering endocytosis. ${ }^{30}$

The ionic interaction between $\mathrm{CT}$ and some anionic polysaccharides, such as $\mathrm{CS},{ }^{14,31} \mathrm{FC},{ }^{32}$ and heparin, ${ }^{33,34}$ has recently been used to produce micro- (CS) or nanoparticles (heparin) with biological activity. The production of these micro- (CS) and nanostructured materials is feasible through ionic interactions among the polyelectrolytes, ie, the positive charges of $\mathrm{CT}$ at acid $\mathrm{pH}$ interacting with the negatively charged sulfated polysaccharides (interpolymer complexation). Many scientific reports, including those from Calvo et $\mathrm{al}^{35}$ have pointed to NP production using biodegradable hydrophilic polymers. In these works, a CT NP production method using the ionic interaction with sodium tripolyphosphate for protein delivery was described. Thus, the positive charges of CT amino groups interact with negatively charged tripolyphosphate, forming particulate material in the nanometer range.

NP production through ionic interaction between polyelectrolytes, such as CT and TP, has been previously described. ${ }^{36}$ The literature also includes the use of CT and dextran for the release of doxorubicin; ${ }^{28} \mathrm{CT}$ and heparin ${ }^{33,34}$ for gene therapy, and CT and alginate ${ }^{37}$ and CT and gelatin for the controlled release of cyclosporine-A. ${ }^{29}$

The aim of this work was to develop a new nanocoacervation method in order to obtain sulfated polysaccharide NPs with anticoagulant activity. The optimization of the anticoagulant activity of these polysaccharides in nanoparticulate systems, which restricts the conformation freedom of these macromolecules, was evaluated by different approaches. Furthermore, the feasibility of preparing CT NPs with different natural polyanions, as a promising oral anticoagulant formulation, was studied.

\section{Materials and methods Materials}

Chitosan of low molar weight/deacetylation degree $\geq 90 \%$ (Sigma, St. Louis, USA), fucoidan from Fucus vesiculosus (Sigma, St. Louis, USA), chondroitin-4-sulfate (Sigma, St Louis, USA), sodium borate $\left(\right.$ Borax $^{\circledR}$ ) (Merck, Darmstadt, Germany), and acetic acid (Vetec, Rio de Janeiro, Brazil) were used in analytical grade. Milli-Q water (Millipore, USA) was used in the preparation of all buffers and solutions.

\section{Methods}

\section{Preparation of polysaccharide nanoparticles by nanocoacervation}

In this work, methods previously described in the literature were adapted for a regular coacervation process. ${ }^{5,14,38-40}$ Briefly, CT was used as the positive (cationic) and CS or FC as negative (anionic) polyelectrolytes. Then, $30 \mathrm{~mL}$ of a solution of the negative polyelectrolyte in borax buffer or water were dripped $(30 \mathrm{~mL} / \mathrm{h})$, using a syringe-based droplet system, onto $30 \mathrm{~mL}$ of $\mathrm{CT}$ in acetic acid $1 \%(\mathrm{v} / \mathrm{v})$, under magnetic stirring and sonication at $100 \%$ intensity in an ice bath for 60 minutes. The distance between the needle and the CT solution was $10 \mathrm{~cm}$. The polyelectrolyte concentrations tested in this work are presented in Table 1. After complete dripping, dispersions were centrifuged at $548.51 \times \mathrm{g}$ for 30 minutes in a SIGMA 4-16 KH refrigerated centrifuge (Osterode, Germany), the pellet was separated, and the supernatant was centrifuged at $28435.21 \times \mathrm{g}$ for 30 minutes. After separating the supernatant, the pellet was resuspended 
Table I Combination of polysaccharide solutions to produce four different NP suspensions

\begin{tabular}{lllll}
\hline Sample & Solvents & CT (I\% acetic acid) & CS & FC \\
\hline NPI & Borax buffer & $0.1 \%$ & $0.1 \%$ & \\
NP2 & Borax buffer & $0.1 \%$ & & $0.1 \%$ \\
NP3 & Water & $0.1 \%$ & $0.1 \%$ & \\
NP4 & Water & $0.1 \%$ & & $0.1 \%$ \\
NP5 & Borax buffer & $0.05 \%$ & $0.05 \%$ & \\
NP6 & Borax buffer & $0.05 \%$ & & $0.05 \%$ \\
NP7 & Water & $0.05 \%$ & $0.05 \%$ & \\
NP8 & Water & $0.05 \%$ & & $0.05 \%$ \\
\hline
\end{tabular}

Abbreviations: CS, chondroitin sulfate; CT, chitosan; FC, fucoidan; NP, nanoparticle.

in $1000 \mu \mathrm{L}$ of water and used in the following measurements. All experiments were performed in triplicate.

\section{Mean diameter and polydispersity index measurements}

Mean diameter (MD) and polydispersity index (PI) measurements were conducted on a Horiba LB-550 DLS analyzer (Kyoto, Japan), with a detection angle of $90^{\circ}$, 100 scans over 2 minutes each for each sample, with a refraction index adjusted to 1.33 and a temperature of $22^{\circ} \mathrm{C}$. These measurements were performed in triplicate for each NP sample, on different days for different solutions.

\section{Determination of zeta potential}

The ZP was measured using Malvern nanosizer software (Worcestershire, United Kingdom). A mean value was obtained from three readings for each sample. The parameter values $-\mathrm{pH}$, refractive index, and average diameter - were imputed in the equipment before analyzing.

\section{Transmission electron microscopy (TEM)}

The samples were maintained for 5 minutes on copper grids of 400 mesh covered with formvar film, sprayed with carbon. The grids were dried with filter paper and contrasted in aqueous uranyl acetate 5\% for 1 minute, and then observed under a FEI Morgagni (Hillsboro, OR) microscope operating at $80 \mathrm{kV}$.

\section{Study of particle separation by centrifugation}

The NP dispersions were centrifuged at increasing speeds, in order to observe size distribution obtained at each speed used and suitability of NP purification. The following speeds were tested: 87.76, 548.51, 2194.07, $4936.66,19746.67$, and $28435.21 \times \mathrm{g}$. The six pellets were collected and resuspended in $500 \mu \mathrm{L}$ of water by sonication for 30 minutes. The MD of the resuspended pellet and the supernatant were measured.

\section{Sulfated polysaccharide assays}

NP samples isolated by centrifugation were mixed with different acidic solutions $(10 \%, 50 \%$, and $100 \%$ acetic acid; $0.1 \mathrm{M}$ and $1 \mathrm{M}$ hydrochloric acid) at $25.0 \mu \mathrm{g} / \mathrm{mL}$ concentration in order to obtain the complete dissolution of polyelectrolyte complex. The dissolution was confirmed by DLS analysis. These samples were complexed with dimethylmethylene blue (DMB) and evaluated by spectrophotometry at $525 \mathrm{~nm} .{ }^{41}$ The concentration of sulfated polysaccharide in the NP samples was calculated using a calibration curve obtained with $\mathrm{FC}$ at $1.0,5.0,10.0,20.0,30.0$, and $40.0 \mu \mathrm{g} / \mathrm{mL}$, in triplicate.

\section{Coagulation tests}

The ability of FC NPs to potentiate inhibition of rat plasma coagulation was assessed by measuring NP2 recalcification time in an Amelung KC4 A coagulometer (Mount Holly, NJ). Citrated blood samples were centrifuged $(2000 \times \mathrm{g})$ for 10 minutes, and the platelet-poor plasma was stored at $-20^{\circ} \mathrm{C}$ until use. Pre-warmed rat plasma (100 $\mu \mathrm{L}$ containing 3.8\% sodium citrate; $1: 9, \mathrm{v} / \mathrm{v}$ ) was incubated with NP2 samples at various concentrations (suspension in saline solution) for 1 minute at $37^{\circ} \mathrm{C}$. The addition of $100 \mu \mathrm{L}$ of pre-warmed $50 \mathrm{mM} \mathrm{CaCl}_{2}$ started plasma clotting. The coagulation time was recorded in seconds. ${ }^{42-44}$

Additional assays were performed to verify the NP anticoagulant effects on activated partial thromboplastin time (aPTT) and prothrombin time (PT). For aPTT tests, cephalin plus kaolin (aPTT reagent) was incubated for 1 minute with $50 \mu \mathrm{L}$ of pre-warmed plasma $\left(37^{\circ} \mathrm{C}\right)$ and selected $\mathrm{NP}$ samples, at various concentrations. The reaction was started by the addition of $100 \mu \mathrm{L}$ of pre-warmed $\mathrm{CaCl}_{2}(50 \mathrm{mM})$. For PT tests, $50 \mu \mathrm{L}$ of pre-warmed rat plasma was incubated with NP samples, at various concentrations, for 2 minutes, at $37^{\circ} \mathrm{C}$. The reaction was started by the addition of $100 \mu \mathrm{L}$ of pre-warmed thromboplastin with calcium (PT reagent). ${ }^{42-44}$ Only FC NPs were tested and compared to free polysaccharide solution, as the effects of anticoagulation for CS are not reported in the literature.

\section{Cytotoxicity test for evaluating polysaccharides Caco-2 cell toxicity}

Caco-2 cells were seeded in 96-well plates (CELLSTAR ${ }^{\circledR}$; Greiner Bio-One GmbH, Frickenhausen, Germany) at a density of 32,000 cells/well, distributed in a total volume of $200 \mu \mathrm{L} / w e l l$. Then, the plates were taken to a Galaxy CO2 cell incubator (New Brunswick Scientific, Enfield, CT) at $37^{\circ} \mathrm{C}$ and 5\% CO2 for 24 hours. After incubation, the cells were placed in contact with the following samples: FC solutions 
in HBSS $\mathrm{pH}=6.8(500,1000$, and $1500 \mathrm{mg} / \mathrm{mL})$, physical mixtures of CT and FC in HBSS pH $=6.8$ (250, 500, and $1000 \mathrm{mg} / \mathrm{mL}$ ), and NPs of CT and FC resuspended in Hank's Balanced Salt Solution (HBSS) $\mathrm{pH}=6.8$ (250, 500, and $1000 \mathrm{mg} / \mathrm{mL}$ ).

HBSS $\mathrm{pH}=6.8$ was used as a positive control, and a negative solution $10 \%$ Tryton X-100 (Tryton ${ }^{\circledR}$ X-100 Merck, Darmstadt, Germany). The samples were kept in contact with the cells for 3 hours and then aspirated and treated with MTT [3-(4,5-Dimethylthiazol-2-yl)-2,5-diphenyl tetrazolium bromide] reagent $(2.5 \mathrm{mg} / \mathrm{mL})$ by adding $100 \mathrm{~mL}$ of HBSS and $25 \mathrm{~mL}$ of MTT solution per well. The plates containing the cells were incubated with MTT for 3 hours at $37^{\circ} \mathrm{C}$ and $5 \% \mathrm{CO}_{2}$. At the end of this incubation time, MTT was aspirated, and the cells were washed with phosphate buffer solution $(\mathrm{PBS})(\mathrm{pH}=7.4)$. The PBS was then aspirated, and $100 \mathrm{~mL} /$ well of dimethyl sulfoxide (DMSO) (Sigma Aldrich, Milano, Italy) was added, in order to break the cell membrane. That allowed the release of formazam crystals formed, generating a purple color more or less intense according to the degree of cell viability. The absorbance readings were performed in wells of Microplate Absorbance Reader iMARK ${ }^{\mathrm{TM}}$ (Bio-Rad Laboratories Srl, Segrate, Italy), with reference to $570 \mathrm{~nm}-690 \mathrm{~nm}$, after shaking vigorously for 60 seconds. ${ }^{45}$

\section{Permeability studies performed by means of Caco-2 cell monolayer}

The nanoparticle suspensions (NP2) prepared in $\mathrm{pH} 6.8$ HBSS were subjected to permeability tests across Caco-2 cell monolayers. Caco-2 cells (passage 38) were seeded on tissue-culture-treated polycarbonate filters (area $113.1 \mathrm{~mm}^{2}$; inner diameter $13.85 \mathrm{~mm}$ ) in 12-well plates (Greiner Bio-one, International PBI, Italy) at a seeding density of $1 * 10^{5}$ cells $/ \mathrm{cm}^{2}$. Dulbecco's Modified Eagle's Medium (DMEM, $\mathrm{pH}$ 7.40; Bio Industries, Israel), supplemented with 10\% fetal bovine serum, benzylpenicillin $\mathrm{G}(160 \mathrm{U} / \mathrm{mL})$, and streptomycin sulfate $(100 \mu \mathrm{g} / \mathrm{mL})$ (Bio Industries) as well as with $1 \%$ nonessential amino acids (Sigma, I), was used as culture medium. Cell cultures were kept at $37^{\circ} \mathrm{C}$ in an atmosphere of $95 \%$ air and 5\% CO2 and 95\% of relative humidity. Filters were used for transepithelial electrical resistance (TEER) measurements and transport experiments 21-23 days after seeding. ${ }^{45}$

Five hundred microliters of FC solution or NP2 suspension, prepared as previously described in the "Preparation of polysaccharide nanoparticles by nanocoacervation" paragraph, at $1000 \mu \mathrm{g} / \mathrm{mL}$ in HBSS at $\mathrm{pH}$ 6.8, was used as the apical (donor) phase of the monolayers. HBSS at $\mathrm{pH} 7.4$ (1.5 mL) was used as the basolateral (receptor) phase and added to the basolateral side of the monolayers. At 30,60, 120, and 180 minutes, each filter and its mounting donor chamber, filled with the apical phase, was moved into a fresh basolateral (receptor) phase. All the receptor phases were collected, and the permeated sulfated polysaccharides were assayed by means of the spectrophotometric method previously described.

During the experiments and until 7 hours afterward, the integrity of the monolayers was assessed by means of TEER measurements at fixed times, using a Millicell ERS-meter (Millipore Corp., Bedford, MA).

\section{Statistical data analysis}

Statistical differences were determined using SigmaPlot 11.0 (Systat Software, Inc). Differences between groups were considered significant at $P<0.05$.

\section{Results and discussion}

The NP preparation method was based on the ionization of CS and FC polysaccharides carboxyl and sulfate groups, which interact efficiently with the positively charged CT under controlled sonication. This method did not use surfactants or organic solvents, only magnetic stirring and sonication to break the millimetric drop and form nanometric spherical particles. Another positive aspect of the present method was the droplet control with a fixed value and controlled distance between the needle and the CT solution, which avoided the undesirable variation of these parameters, making the scale-up method more feasible. The developed method in the present work was less expensive and cleaner than other techniques in the literature..$^{5,14,38-40}$ A similar procedure was proposed by Chen et al (2009); ${ }^{33}$ however, the NPs were prepared exclusively by heparin solution drop, using a pipette, into a CT solution, without sonication. These results indicated a close relationship between the heparin structure and NP formation, indicating a limited applicability of this polysaccharide. The formulations presented in Table 1 did not show agglomeration or follow the characterization. The $\mathrm{pH}$ measurements of each polysaccharide solution and the nanometric dispersions were carried out in order to optimize the preparation process and to evaluate the impact of $\mathrm{pH}$ on the anticoagulant and antithrombotic activity. The $\mathrm{pH}$ values of $0.05 \%$ and $0.1 \% \mathrm{CS}$ or FC solutions in borax buffer were between 8.9 and 9.1; for sulfated polysaccharides in water, at the same concentration, the observed $\mathrm{pH}$ values were between 6.2 and 6.6. The CT solution $0.05 \%$ and $0.1 \%$ 
in acetic acid presented $\mathrm{pH}$ values between 2.8 and 3.1. NP dispersions prepared with borax buffer solutions presented $\mathrm{pH}$ values between 3.9 and 4.1, whereas those prepared with water presented $\mathrm{pH}$ values between 2.5 and 2.7. The results showed that the ionization of only one of the biopolymers using a buffer could lead to coacervate formation. This justifies the procedure described by Chen et al (2009), ${ }^{33}$ wherein a coacervation was obtained by mixing ionized $\mathrm{CT}$ solution with heparin dissolved only in water. However, to ensure greater efficiency in the process studied herein, NPs were prepared using buffer in both polysaccharides.

The $\mathrm{pH}$ of the NP suspensions indicated the need for centrifugation and washing the material, followed by redispersion, to carry out the testing of in vitro pharmacological activity, to avoid any interference in the obtained results. The highest concentrations of polysaccharides used $(0.1 \%$ $\mathrm{w} / \mathrm{v}$ ) were similar to those normally used for NP preparation on a laboratory scale. Despite the simplicity of the process, scaling up was not considered in this study, as the goal was to focus on evaluating the effect of the nanostructuring of polysaccharides in their pharmacological activity, and not in the industrialization of the product.

The MD, PI, and ZP values for proposed formulations are shown in Table 2. Np1 and Np7 showed the lowest and the highest MD, $154.2 \pm 35.77 \mathrm{~nm}$ and $453.37 \pm 369.48 \mathrm{~nm}$, respectively. According to Gaumet et al, ${ }^{46}$ smaller NPs are less susceptible to the mononuclear phagocytosis system and present higher circulation times in the organism; therefore, NP1 was selected as the best candidate for the CS carrier, compared to NP3, NP5 and NP7. Despite having sizes close to that of NP1 $(P=0.0034)$, other nanosystems containing CS showed higher PI and lower reproducibility. NP2 was selected as the best nanocarrier for FC, compared to NP4, NP6, and NP8, for the same reasons applied to NP1.

For FC, an increased variation in particle size distribution could be observed. This was probably due to the molecular

Table 2 Mean diameter, polydispersity index, and zeta potential values for different formulas

\begin{tabular}{llll}
\hline Sample & MD \pm SD $(\mathbf{n m})$ & PI \pm SD & ZP \pm SD $(\mathbf{m V})$ \\
\hline NPI & $154.20 \pm 35.77$ & $0.109 \pm 0.015$ & $34.5 \pm 0.65$ \\
NP2 & $198.00 \pm 38.84$ & $0.139 \pm 0.019$ & $43.3 \pm 0.48$ \\
NP3 & $189.63 \pm 17.57$ & $0.149 \pm 0.020$ & $41.2 \pm 0.74$ \\
NP4 & $352.73 \pm 41.46$ & $0.203 \pm 0.011$ & $52.5 \pm 1.00$ \\
NP5 & $180.90 \pm 63.04$ & $0.207 \pm 0.044$ & $35.88 \pm 1.74$ \\
NP6 & $269.80 \pm 201.78$ & $0.262 \pm 0.031$ & $35.77 \pm 3.12$ \\
NP7 & $453.37 \pm 369.48$ & $0.265 \pm 0.022$ & $44.94 \pm 0.85$ \\
NP8 & $341.70 \pm 200.00$ & $0.257 \pm 0.059$ & $46.60 \pm 0.67$ \\
\hline
\end{tabular}

Notes: Mean values $\pm \mathrm{SD} ; \mathrm{n}=3$. Abbreviation: SD, standard deviation. weight distribution being narrower in CS compared to FC, which did not have the fractions of mass separated in order to simulate a more accurate use of the crude natural product as a new drug. The best experimental result was obtained with NP2, which showed an adequate mean and SD of size distribution, PI, and ZP $(P=0.0021)$. The lowest MD and PI were reached using $0.1 \%$ of polysaccharides solutions in borax buffer (ie, NP1 and NP2) (Table 2), indicating the possibility of obtaining a better result with an increased concentration of polysaccharide.

Similar results were observed for all samples using borax buffer solutions (NP1, NP2, NP5, and NP6), which presented lower MD and ZP values than those with water (NP3, NP4, NP7, and NP8). These profiles might be due to the alkaline medium solutions, as acidic polysaccharides are less ionized in neutral solvent, indicating the need for ionization of both polysaccharides to improve the preparation of NPs.

The literature has reported the production of CT NPs with tripolyphosphate by using coacervation with copolymer insertion, such as polyethylene. The MDs obtained for these NPs were between 263.8 and $745.5 \mathrm{~nm} .{ }^{35}$ Apparently, the dripping method performed by these authors presents a higher $\mathrm{MD}$ and also increases the cost, mainly due to the use of specific reagents, in comparison to our new methodology. The low polydispersity values obtained for NP1 $(0.109 \pm 0.015)$ and NP2 $(0.139 \pm 0.019)$ revealed that samples with $0.1 \%$ of $\mathrm{CS}$ or $\mathrm{FC}$ in alkaline medium are more homogenous for MD particles. However, systems obtained using 0.05\% polysaccharide solutions did not show the same features. All systems showed positive superficial electrical density. NP samples using borax buffer solution had lower ZP values than those with water. NP1 showed a lower ZP value than NP5 and NP7, and almost the same value as NP3, suggesting that polysaccharide concentration is a non-interfering factor. However, FC NPs did not show the same ZP values for different concentrations, as can be seen in NP2 and NP6. All zeta potential values suggest MD stability for the NPs, as they had no neutral values, avoiding particle aggregation. Low-ZP NPs should produce the best pharmacological profiles, due to a reduction of opsonization.

The literature reports chitosan-heparin NP formation to produce a carrier for bovine serum albumin, using similar polysaccharide concentrations, low molecular weight chitosan, and a dropwise technique. The authors reported values of $204.4 \mathrm{~nm}, 0.189$, and $+29.39 \mathrm{mV}$ for MD, PI, and $\mathrm{ZP}$, respectively. ${ }^{47}$ Sezer et $\mathrm{al}^{32}$ produced fucospheres by using polyelectrolyte complexation between CT and FC. In this report, the smaller fucosphere showed a larger mean 
diameter than NP2, as well as a higher PI and lower ZP than produced in this study. Those authors did not evaluate the anticoagulant activity of their fucospheres, but rather, their efficacy in the treatment of dermal burns. ${ }^{32}$ Compared with the previously described chitosan-heparin NP preparation with anticoagulant activity, ${ }^{33}$ no similar results or reproducibility were obtained for our FC and CS NP preparation, with sulfated polysaccharide incorporation yield into the NPs ranging between $25 \%$ and $70 \%$. Characteristic parameters for regression equation $(y=0.0096 \mathrm{X}+0.0046)$ of the DMB sulfated polysaccharide determination method obtained by least squares treatment of the results confirmed the good linearity of the method developed $(r=0.9953)$. In that regard, five-sample replicates were consecutively tested, using the same equipment, at a concentration of $25 \mu \mathrm{g} / \mathrm{mL}, 100 \%$ of the normal analytical working value.

The NP1 TEM data presented in Figure 1(A and C), show the presence of NPs with spherical or oval form. There are no significant particle aggregates, and the MD value is close to that observed in the DLS analysis. Figure 1(B and D) also shows particles in the $200 \mathrm{~nm}$ range, which reinforces the MD data measured by DLS analysis. The NP2 system was also submitted to TEM (Figure 2), which showed NPs with spherical shape and MD in the same order as DLS analysis.

To analyze the effects of coacervation in the shapes of the NPs obtained and compare the sizes of the nonnanostructured material that could agglomerate in vivo, the physical mixture of the polysaccharides was evaluated by TEM analysis. The physical mixture of the polysaccharides $(\mathrm{CT}+\mathrm{CS}$ and $\mathrm{CT}+\mathrm{FC})$ was submitted to common stirring

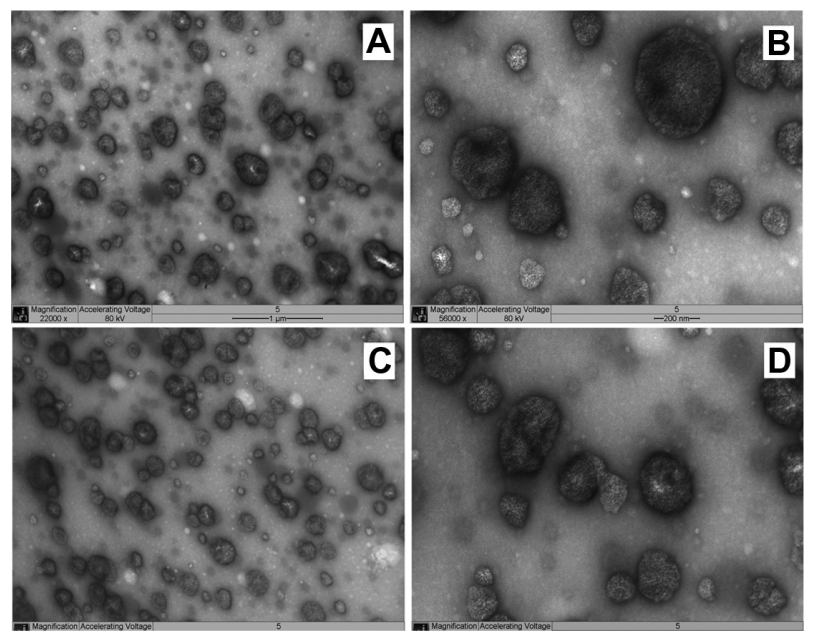

Figure I NPI TEM images of two different points ( $\mathbf{A}$ and $\mathbf{C})$ of the same sample $(22000 \times)$. Images (B and D) shows higher magnification (56000x) of (A and $\mathbf{C})$, respectively.

Abbreviations: NP, nanoparticle; TEM, transmission electron microscopy.

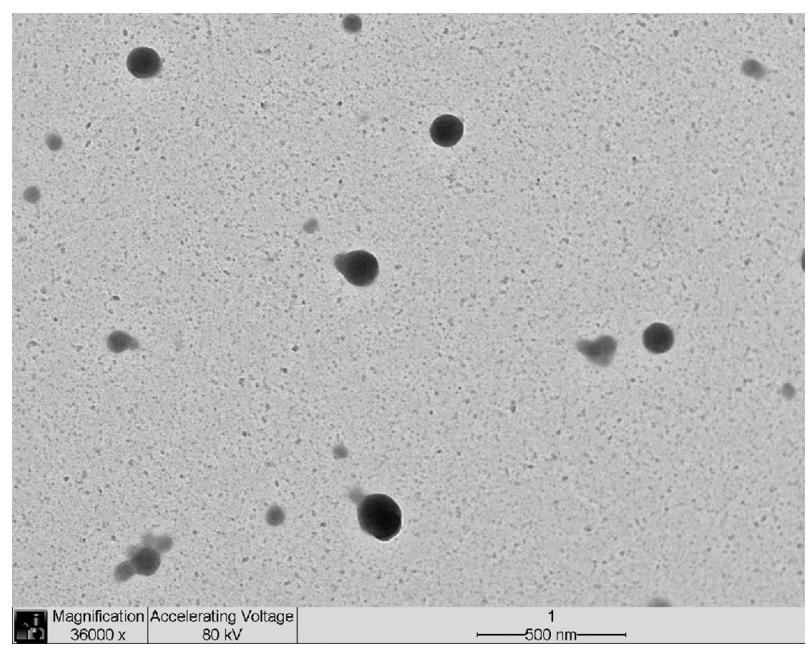

Figure 2 NP2 TEM image (36000× magnification).

Abbreviations: NP, nanoparticle; TEM, transmission electron microscopy.

and sonication. According to TEM presented in Figure 3 (A and $\mathrm{B}$ ), the physical mixture morphology was not well defined in this experiment, in contrast to NP1 and NP2, produced by the nanocoacervation method (Figures 1 and 2), with MDs quite similar to those observed for NP1 and NP2. The morphology difference between NP structure and physical mixture arrangement showed that, without sonication, there is no nanosphere formation.

The particle analysis performed on NP1 after sequential centrifugation revealed particles presenting MD of $3545.8 \mathrm{~nm}(>5.0 \%$ of total mass) for NP1 at the first step. The supernatant was sequentially centrifuged; the decrease in MD in the NP1 particles is shown in Figure 4. The NP1 MD decreased to $185.4 \mathrm{~nm}$ after centrifugation at $28435.21 \times \mathrm{g}$. This result suggests that lower particles may be separated using a higher speed level and using as ideal conditions the previous centrifugation at $548.51 \times \mathrm{g}$, followed by $28435.21 \times \mathrm{g}$. The main purpose is to discard the major particles and test those with MDs between 100 and $400 \mathrm{~nm}$. This was necessary to avoid high PI and to ensure that the results of anticoagulant activity are due to the NPs and not to the

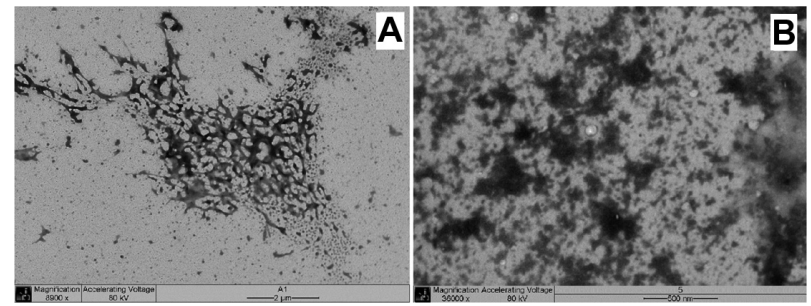

Figure 3 Physical mixtures TEM. (A) Chondroitin sulfate-chitosan mixture; (B) Fucoidan-chitosan mixture.

Abbreviation: TEM, transmission electron microscopy. 


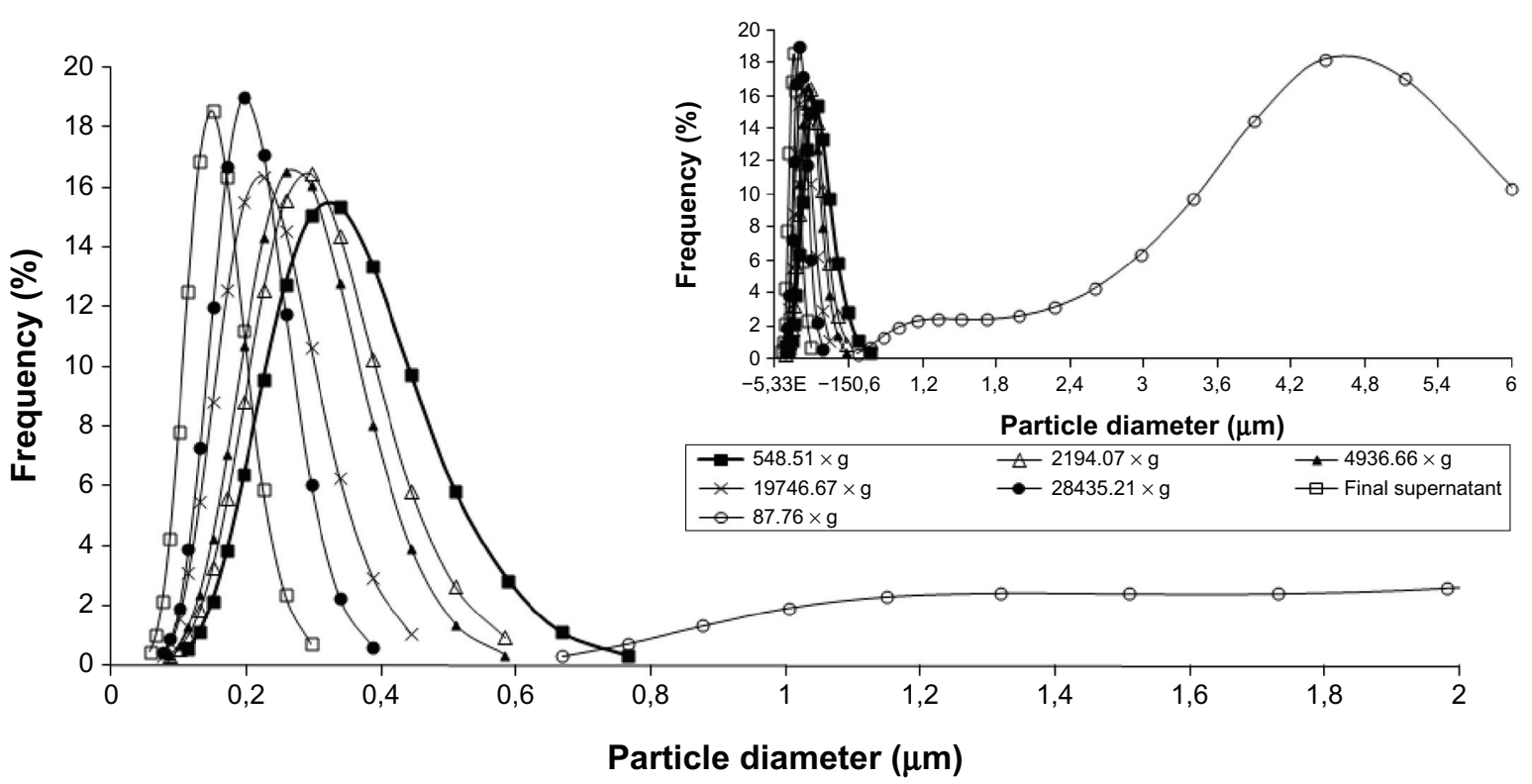

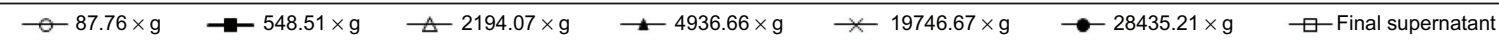

Figure 4 Study of particle separation by centrifugation of NPI

Abbreviation: NP, nanoparticle.

soluble biopolymer. Similar results were observed with NP2 particle separation (Figure 5). However, the distribution was more heterogeneous than that of NP1, for the reasons already presented. Therefore, a separation procedure identical to that of NP2 was established for NP1. Centrifugation was a good choice, as it is faster than dialysis, less expensive than gel filtration, and less aggressive to polysaccharide NP structures than lyophilization. ${ }^{48}$ However, the very similar results of PI measurements indicate that the use of unseparated NPs does not represent a problem for pharmacological performance of these new anticoagulant formulations. Therefore, unseparated FC-NP preparations and FC solution were selected and tested in the coagulation assays.

Thus, NP2 and NP1 were selected for the subsequent tests. DLS analysis showed that chloride acid $1 \mathrm{M}$ solution was able to break NP2 containing sulfated polysaccharides, resulting in a solution with a $5.4 \mathrm{~nm}$ MD. Other acid solutions could not break the NPs with high MD values and similar diameters. Similar results were obtained for NP1. The soluble FC and CS were quantified by $\mathrm{DMB}$ - metachromatic assay. The $\mathrm{FC}$ content in resultant NP2 samples was $75.85 \pm 0.919 \mu \mathrm{g} / \mathrm{mL}(\mathrm{n}=3)$, and $\mathrm{CS}$ content was $25.16 \pm 0.817 \mu \mathrm{g} / \mathrm{mL}(\mathrm{n}=3)$. Fucoidan yield in NP2 was similar in these NPs, as reported by Chen et al (2009) ${ }^{33}$ in chitosan-heparin NPs, which used a different polymer.

The in vitro coagulation tests confirmed the anticoagulation activity of the NPs compared to the control, using PBS for NP2 (FC NP), with no significant results for NP1 $(\mathrm{CS} \mathrm{NP}) ; P=0.00345$.
For NP2, in the aPTT test, the coagulation time $(13.3 \mu \mathrm{g} / \mathrm{mL})$ was two times higher than that of the control (Figure 6), whereas sulfated polysaccharide free solution increased only 1.6 times ( $40 \%$ lower). The NP dispersion $\mathrm{pH}$ was about 4.5 , not indicating an effect in in vitro coagulation tests. FC solution $(2.67 \mu \mathrm{g} / \mathrm{mL})$ did not affect the coagulation time compared to the control. On the other hand, NP2 increased the coagulation time, even at a low concentration $(0.53 \mu \mathrm{g} / \mathrm{mL})$ compared to the control $(1.6 \mathrm{x})$. The results of the recalcification tests (Figure 7) were similar to aPTT, with NP2 activity higher than FC solution. NP2 and FC solution $(13.3 \mu \mathrm{g} / \mathrm{mL})$ increased the coagulation time 1.51 and 1.24 times, respectively. In contrast, the prothrombin time (PT) showed no significant difference on coagulation time compared to the control (data not shown). The absence of an activity profile of NP2 and FC solution in PT suggested that the extrinsic pathway is not affected by these substances. Considering the aPTT data, the intrinsic coagulation pathway is the main target of both NP2 and FC solution. These aPTT and PTT results reinforced previous works that showed sulfated polysaccharides in free form affecting the coagulation intrinsic pathway. ${ }^{44}$

NP2 showed higher anticoagulant activity than FC solution, possibly due to the fact that polysaccharides in solution may assume a nonspecific conformation that leads to lower activity, contrasting with NP2, which has a fixed conformation, permitting a more effective receptor interaction and activity. ${ }^{33}$ 


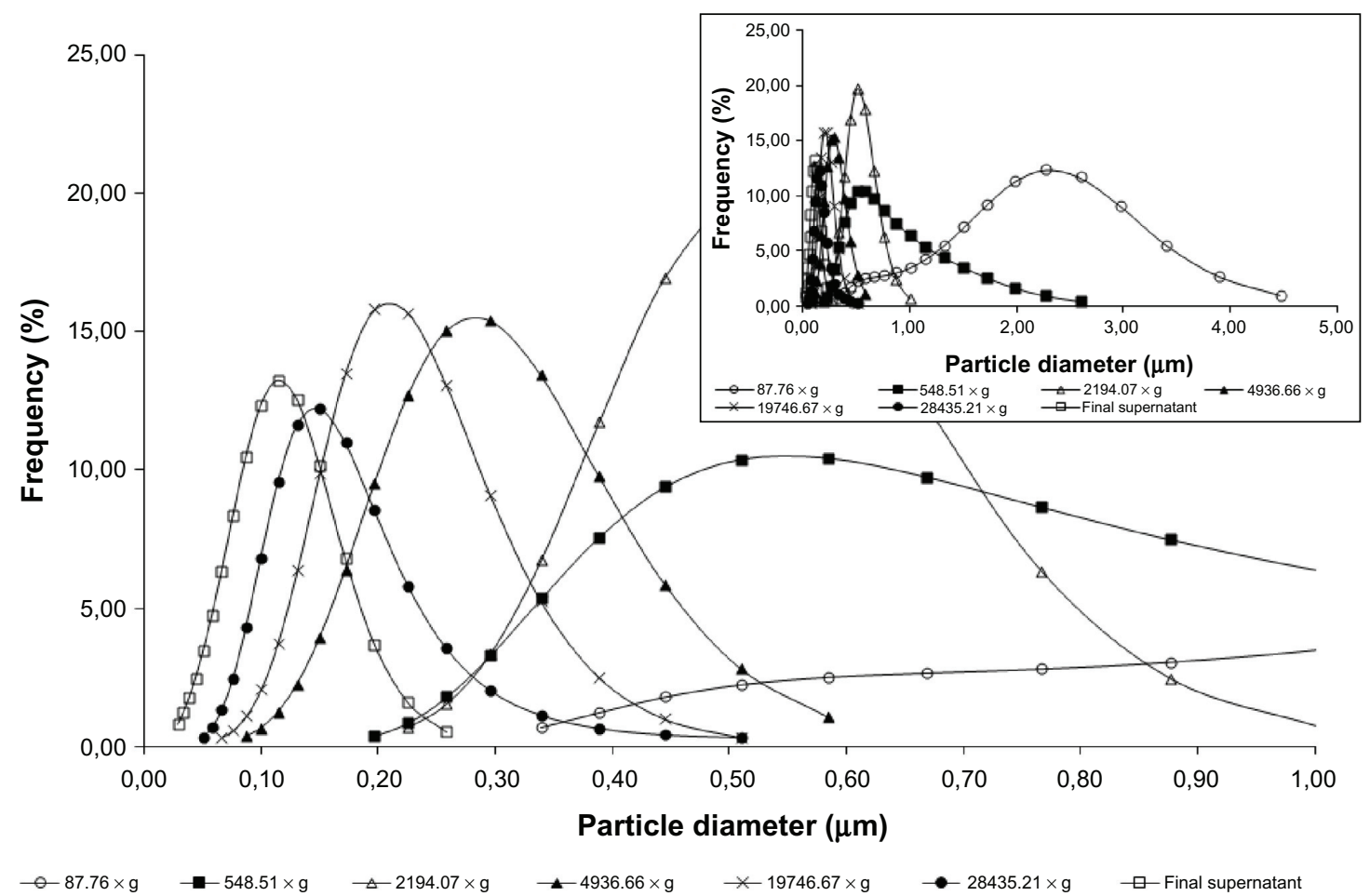

Figure 5 Study of particle separation by centrifugation of NP2.

Abbreviation: NP, nanoparticle.

CS did not exhibit the same anticoagulant properties as FC, but there are some reports in the literature showing antinflamatory ${ }^{49}$ and anticancer activities. ${ }^{50,51}$ For these reasons, these CS NPs can be tested as a new anticancer system with carrier properties. In this work, NP1 was prepared only as a nanocoacervation model for NP2, for economic reasons.

It was necessary to conduct the cytotoxicity assay with MTT in order to verify that the NPs, physical mixtures, and polysaccharide solutions showed some toxicity for the Caco-2 cell monolayer, considering the possible oral administration of the nanostructured systems under study. Cell viability is determined by the capacity of the mitochondrial enzyme succinate dehydrogenase to reduce MTT (tetrazole salt) to formazam. The fucoidan-chitosan physical mixture showed a decrease of cell viability, increasing polymer concentrations from $250 \mathrm{ug} / \mathrm{mL}$ to $2000 \mathrm{ug} / \mathrm{mL}$ and suggesting a cytotoxic effect

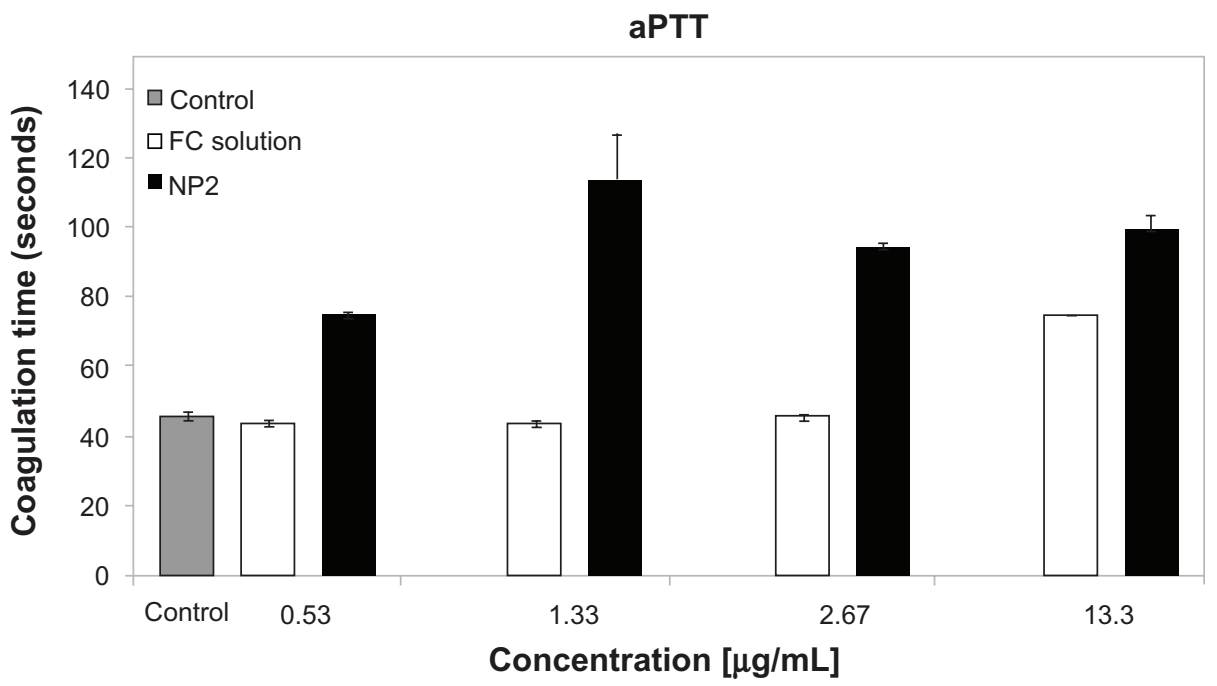

Figure 6 aPTT test for NP2 and FC solution.

Notes: Mean values \pm SD; $\mathrm{n}=3$.

Abbreviations: aPTT, activated partial thromboplastin time; FC, fucoidan; NP, nanoparticle. 


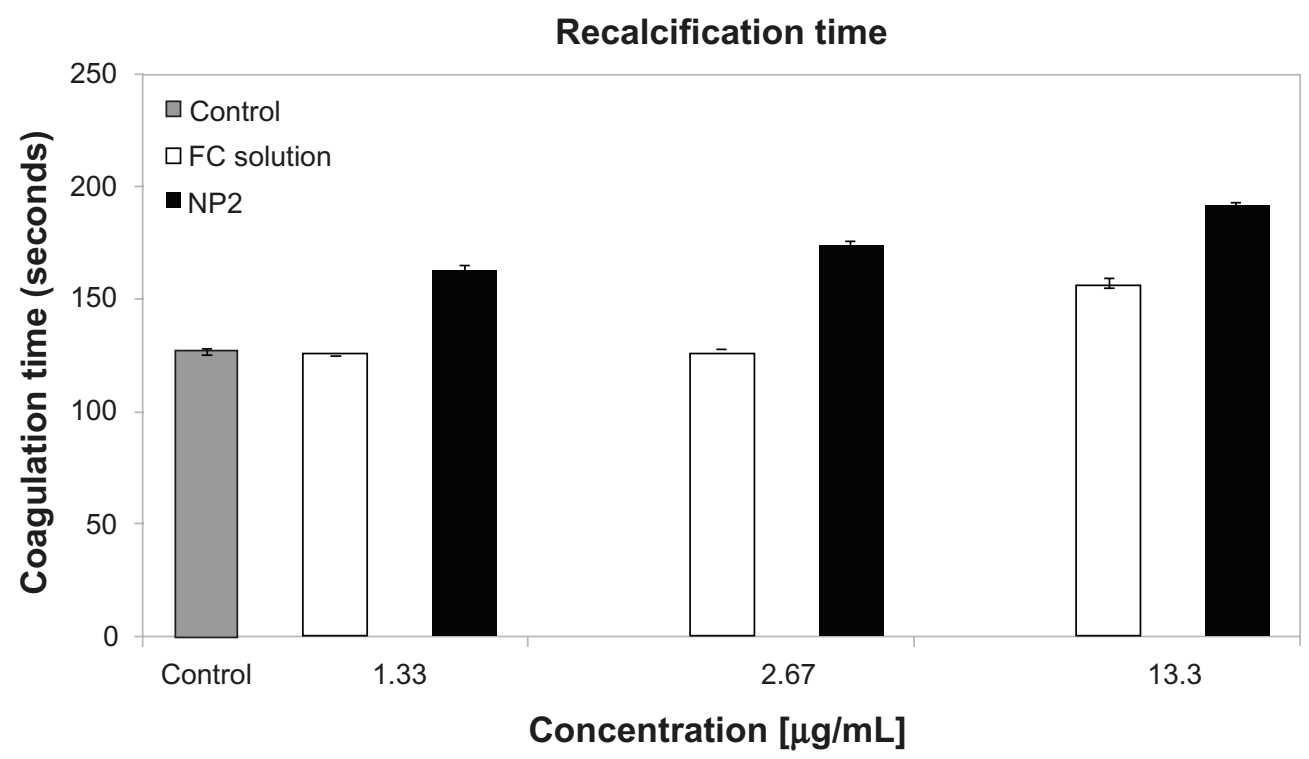

Figure 7 Recalcification test for NP2 and FC solution.

Notes: Mean values $\pm \mathrm{SD} ; \mathrm{n}=3$.

Abbreviations: FC, fucoidan; NP, nanoparticle; SD, standard deviation.

toward Caco-2 cells, probably due to the large average particle diameter, which was able to stop gas and nutritive substance exchanges from the cell substrate. The sulfated polysaccharide solutions in higher concentrations showed no toxicity by keeping the cells fully viable. In the test using fucoidan-chitosan NPs (NP2, 250, 500, and $1000 \mathrm{ug} / \mathrm{mL}$ ) did not show any cytotoxic effect and were biocompatible (Figure 8).

Figure 9 shows TEER\% profiles for tested samples and the control (HBSS pH 6.8). At the beginning of the permeation experiments, the mean TEER value was $503.4 \Omega \mathrm{cm}^{2}$ ( $\mathrm{SD} \pm 47.92 \Omega \mathrm{cm}^{2}$ ), to indicate the junctional integrity of Caco-2 cell monolayers. For HBSS pH 6.8, the TEER \% profile remains almost close to $100 \%$ during the experiment time, indicating that this medium did not impair the permeability barrier of the Caco-2 monolayer in vitro and the paracellular pathway.

The NP2 system showed TEER\% profiles significantly lower than that of the control $(P<0.05)$ in the first 2 hours and not significantly $(P>0.05)$ lower from this time until the end of experiment ( 7 hours). This TEER profile indicates an opening between the cell junctions (TEER decreasing), increasing the Caco-2 monolayer permeability by paracellular pathway, followed by a gradual increase in TEER values (tight junctions closing). These observations indicated that this nanosystem could transiently and reversibly open the tight junctions between Caco-2 cells. This behavior was previously observed in the literature in different $\mathrm{pH}$ ranges, displaying more prominence

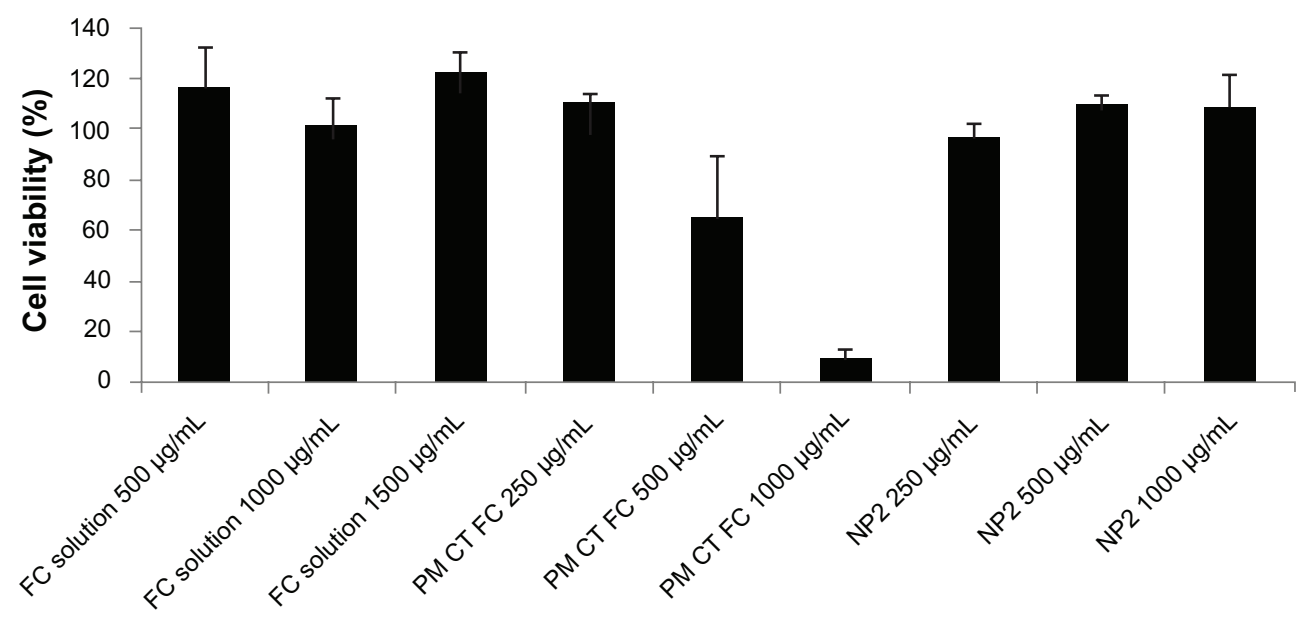

Figure 8 Cytotoxicity test for FC solution, NP2, and physical mixture between FC and CT.

Notes: Mean values $\pm \mathrm{SD} ; \mathrm{n}=3$.

Abbreviations: CT, chitosan; FC, fucoidan; PM, physical mixture; NP, nanoparticle. 


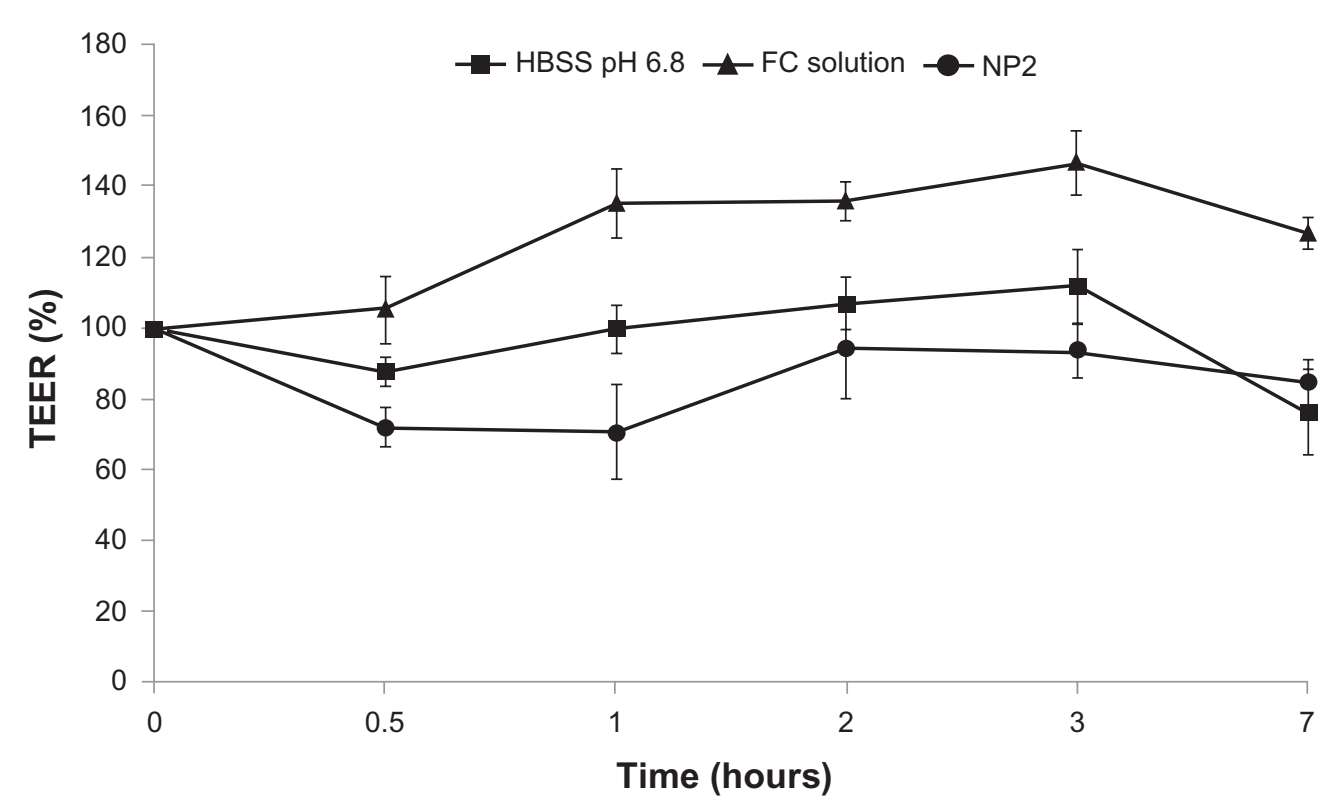

Figure 9 TEER\% vs time profiles observed for NP2, FC solution, and the control (HBSS pH 6.8).

Notes: Mean values $\pm \mathrm{SD} ; \mathrm{n}=3$.

Abbreviations: FC, fucoidan; HBSS, Hank's Balanced Salt Solution; NP, nanoparticle; SD, standard deviation; TEER, transepithelial electrical resistance.

in $\mathrm{pH}$ values under $6.8 .^{33}$ In contrast, the FC solution did not widen the tight junctions, as suggested by TEER values.

The results of sulfated polysaccharides permeation (Figure 10) showed different behaviors for NP2 and FC solution. NP2 presented a higher sulfated polysaccharide permeation percentage from the beginning of the experiment until 120 minutes $(P<0.05)$, compared to the FC solution. During the first hour, NP2 showed two-fold permeation, followed by a slight decrease of this ratio to 1.77 in the second hour. This result suggests higher permeability rates for
NP2 than the corresponding sulfated polysaccharide solution. This could be due to different conformation and dimensions of FC as free polymer chains or in nanoparticulate form. Moreover, since nanoparticle zeta potential was positive due to the presence of chitosan, nanoparticle-cell membrane interactions should be more facilitated with respect to negatively charged free FC molecules. It is conceivable that the size and surface chemistry (zeta potential) of the NPs caused FC absorption via nanoparticle internalization, probably via endocytosis.

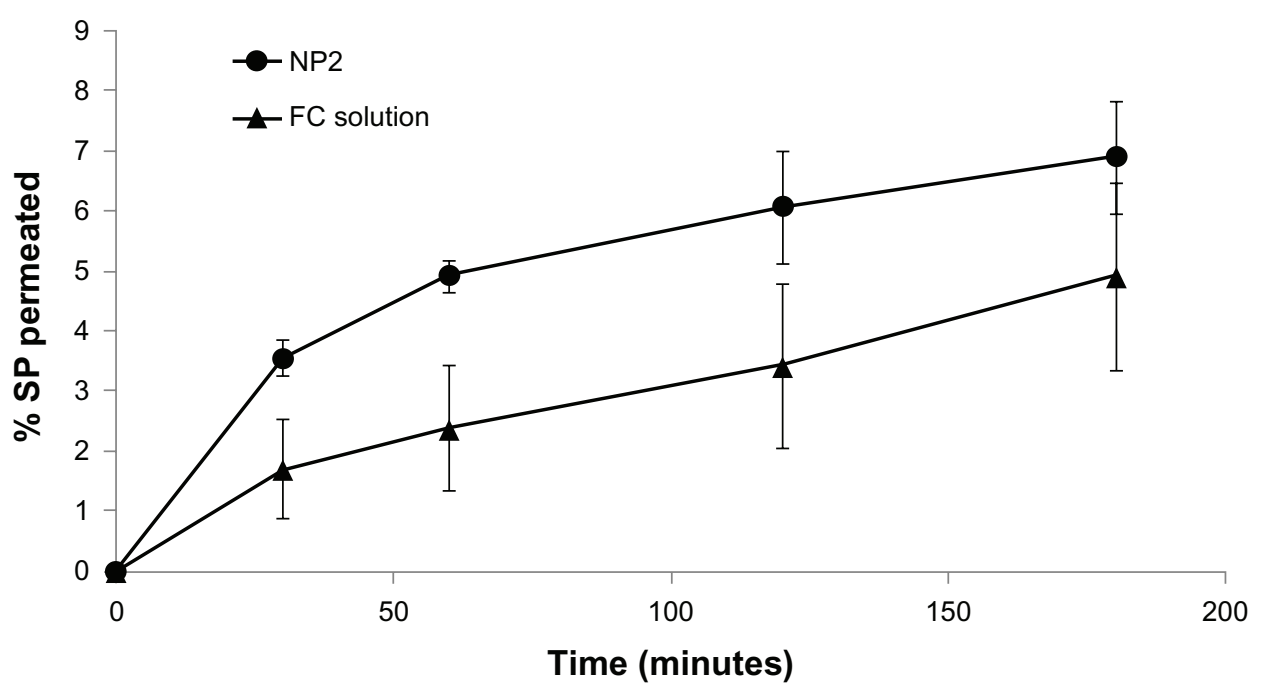

Figure 10 Percentage values of permeated sulfated polysaccharides, calculated for the permeability experiment performed using Caco-2 cell monolayers observed for NP2 and FC solution.

Notes: Mean values $\pm S D ; n=3$.

Abbreviations: FC, fucoidan; NP, nanoparticle; SP, sulfated polysaccharides; SD, standard deviation. 
The particle size was not affect by ionic strength: no particle aggregation occurred in the presence of PBS or HBSS. This is a crucial point, because the particle internalization/endocytosis is strongly hindered by particle propensity to aggregation.

\section{Conclusion}

In this work, we have reported the preparation of sulfated polysaccharide NPs by using a new adapted technique, nanocoacervation, which produces NPs with lower MD and good PI, and uses CS or FC as a therapeutic substance in borax buffer.

The comparison of our systems with those described in the literature containing CT revealed that our system presents a lower MD, with an easier procedure, and that spent fewer reagents, with a cleaner process. The NPs containing $\mathrm{CT}$ and FC presented higher activity than the FC free form at the same concentration in the coagulation tests.

FC-based nanosystems were demonstrated to be biocompatible with Caco-2 cells (intestinal), in view of administration via oral route. Permeability studies across the Caco-2 monolayer showed a greater ability of the NPs to cross the intestinal epithelium when compared to the sulfated polysaccharide solution. This could be due to internalization via endocytosis, due to particle size and surface chemistry (zeta potential). This feature could suggest the possibility of oral administration of such systems.

\section{Acknowledgments}

This work was supported by Coordenação de Aperfeiçoamento de Pessoal de Nível Superior (CAPES) (6711/10-3). Edital CAPES Nanobiotecnologia 2008.

\section{Disclosure}

The authors report no conflicts of interest in this work.

\section{References}

1. Mourao PA, Pereira MS, Pavao MS, et al. Structure and anticoagulant activity of a fucosylated chondroitin sulfate from echinoderm. Sulfated fucose branches on the polysaccharide account for its high anticoagulant action. J Biol Chem. 1996;271(39):23973-23984.

2. Becker CF, Guimaraes JA, Mourao PA, Verli H. Conformation of sulfated galactan and sulfated fucan in aqueous solutions: implications to their anticoagulant activities. J Mol Graph Model. 2007;26(1): 391-399.

3. Mulloy B, Mourao PA, Gray E. Structure/function studies of anticoagulant sulphated polysaccharides using NMR. J Biotechnol. 2000; 77(1):123-135.

4. Oefner PJ, Chiesa C. Capillary electrophoresis of carbohydrates. Glycobiology. 1994;4(4):397-412.

5. Sui W, Huang LL, Wang J, Bo QB. Preparation and properties of chitosan chondroitin sulfate complex microcapsules. Colloids Surf B Biointerfaces. 2008;65(1):69-73.

6. Honda S. Separation of neutral carbohydrates by capillary electrophoresis. J Chromatogr A. 1996;720(1-2):337-351.
7. Park W, Na K. Polyelectrolyte complex of chondroitin sulfate and peptide with lower $\mathrm{pI}$ value in poly(lactide-co-glycolide) microsphere for stability and controlled release. Colloids Surf B Biointerfaces. 2009;72(2):193-200.

8. Bali JP, Cousse H, Neuzil E. Biochemical basis of the pharmacologic action of chondroitin sulfates on the osteoarticular system. Semin Arthritis Rheum. 2001;31(1):58-68.

9. Barkalow FJ, Schwarzbauer JE. Interactions between fibronectin and chondroitin sulfate are modulated by molecular context. J Biol Chem. 1994;269(6):3957-3962.

10. Dace R, McBride E, Brooks K, Gander J, Buszko M, Doctor VM. Comparison of the anticoagulant action of sulfated and phosphorylated polysaccharides. Thromb Res. 1997;87(1):113-121.

11. Mourao PAS, Pereira MS. Searching for alternatives to heparin: sulfated fucans from marine invertebrates. Trends Cardiovasc Med. 1999;9(8):225-232.

12. Kariya Y, Mulloy B, Imai K, et al. Isolation and partial characterization of fucan sulfates from the body wall of sea cucumber Stichopus japonicus and their ability to inhibit osteoclastogenesis. Carbohydr Res. 2004;339(7):1339-1346.

13. Denuziere A, Ferrier D, Domard A. Chitosan-chondroitin sulfate and chitosan-hyaluronate polyelectrolyte complexes. Physico-chemical aspects. Carbohydr Polym. 1996;29(4):317-323.

14. Ganza-Gonzalez A, Anguiano-Igea S, Otero-Espinar FJ, Blanco Mendez JB. Chitosan and chondroitin microspheres for oral-administration controlled release of metoclopramide. Eur J Pharm Biopharm. 1999;48(2):149-155.

15. Muzzarelli RAA, Muzzarelli C. Chitosan chemistry: relevance to the biomedical sciences. Polysaccharides 1: Structure, Characterization and Use. 2005;186:151-209.

16. Rinaudo M. Chitin and chitosan: properties and applications. Prog Polym Sci. 2006;31(7):603-632.

17. Silva HSRC, Santos KSCRd, Ferreira EI. Quitosana: derivados hidrossolúveis, aplicações farmacêuticas e avanços. Química Nova. 2006;29(4):776-785.

18. Liu ZH, Jiao YP, Wang YF, Zhou CR, Zhang ZY. Polysaccharides-based nanoparticles as drug delivery systems. Adv Drug Deliv Rev. 2008;60(15):1650-1662.

19. Oh JK, Lee DI, Park JM. Biopolymer-based microgels/nanogels for drug delivery applications. Prog Polym Sci. 2009;34(12):1261-1282.

20. DudhaniAR, Kosaraju SL. Bioadhesive chitosan nanoparticles: preparation and characterization. Carbohydr Polym. 2010;81(2):243-251.

21. Takeuchi H, Yamamoto H, Kawashima Y. Mucoadhesive nanoparticulate systems for peptide drug delivery. Adv Drug Deliv Rev. 2001;47(1): 39-54.

22. Vasiliu S, Popa M, Rinaudo M. Polyelectrolyte capsules made of two biocompatible natural polymers. Eur Polym J. 2005;41(5):923-932.

23. Koo OM, Rubinstein I, Onyuksel H. Role of nanotechnology in targeted drug delivery and imaging: a concise review. Nanomedicine. 2005;1(3):193-212.

24. Mohanraj V, Chen Y. Nanoparticles - a review. Trop J Pharm Res. 2006;5(1):561-573.

25. Kreuter J. Nanoparticulate systems for brain delivery of drugs. $A d v$ Drug Deliv Rev. 2001;47(1):65-81.

26. Kreuter J. Nanoparticles - a historical perspective. Int J Pharm. 2007;331(1):1-10

27. Kumari A, Yadav SK, Yadav SC. Biodegradable polymeric nanoparticles based drug delivery systems. Colloids Surf B Biointerfaces. 2010;75(1): $1-18$.

28. Mitra S, Gaur U, Ghosh PC, Maitra AN. Tumour targeted delivery of encapsulated dextran-doxorubicin conjugate using chitosan nanoparticles as carrier. J Control Release. 2001;74(1-3):317-323.

29. El-Shabouri MH. Positively charged nanoparticles for improving the oral bioavailability of cyclosporin-A. Int J Pharm. 2002;249(1-2): 101-108.

30. Canton I, Battaglia G. Endocytosis at the nanoscale. Chem Soc Rev. 2012;41(7):2718-2739. 
31. Huang LL, Sui WP, Wang YX, Jiao Q. Preparation of chitosan/ chondroitin sulfate complex microcapsules and application in controlled release of 5-fluorouracil. Carbohydr Polym. 2010;80(1):168-173.

32. Sezer AD, Cevher E, Hatipoglu F, Ogurtan Z, Bas AL, Akbuga J. The use of fucosphere in the treatment of dermal burns in rabbits. Eur $J$ Pharm Biopharm. 2008;69(1):189-198.

33. Chen MC, Wong HS, Lin KJ, et al. The characteristics, biodistribution and bioavailability of a chitosan-based nanoparticulate system for the oral delivery of heparin. Biomaterials. 2009;30(34):6629-6637.

34. Boddohi S, Almodovar J, Zhang H, Johnson PA, Kipper MJ. Layerby-layer assembly of polysaccharide-based nanostructured surfaces containing polyelectrolyte complex nanoparticles. Colloids Surf B Biointerfaces. 2010;77(1):60-68.

35. Calvo P, Remunan Lopez C, Vila-Jato JL, Alonso MJ. Chitosan and chitosan/ethylene oxide-propylene oxide block copolymer nanoparticles as novel carriers for proteins and vaccines. Pharm Res. 1997;14(10):1431-1436.

36. Janes KA, Fresneau MP, Marazuela A, Fabra A, Alonso MJ. Chitosan nanoparticles as delivery systems for doxorubicin. J Control Release. 2001;73(2-3):255-267.

37. Douglas KL, Piccirillo CA, Tabrizian M. Effects of alginate inclusion on the vector properties of chitosan-based nanoparticles. J Control Release. 2006;115(3):354-361.

38. De SJ, Robinson D. Polymer relationships during preparation of chitosan-alginate and poly-1-lysine-alginate nanospheres. J Control Release. 2003;89(1):101-112.

39. Sarmento B, Ribeiro A, Veiga F, Ferreira D. Development and characterization of new insulin containing polysaccharide nanoparticles. Colloids Surf B Biointerfaces. 2006;53(2):193-202.

40. Lee HE, Lee MJ, Park CR, et al. Preparation and characterization of salmon calcitonin-sodium triphosphate ionic complex for oral delivery. J Control Release. 2010;143(2):251-257.

41. Lee CT, Kung PH, LeeYD. Preparation of poly(vinyl alcohol)-chondroitin sulfate hydrogel as matrices in tissue engineering. Carbohydr Polym. 2005;61(3):348-354.
42. Costa LS, Fidelis GP, Cordeiro SL, et al. Biological activities of sulfated polysaccharides from tropical seaweeds. Biomed Pharmacother. 2010;64(1):21-28.

43. Mendes-Silva W, Assafim M, Ruta B, Monteiro RQ, Guimaraes JA, Zingali RB. Antithrombotic effect of Glycyrrhizin, a plant-derived thrombin inhibitor. Thromb Res. 2003;112(1-2):93-98.

44. Nishino T, Nishioka C, Ura H, Nagumo T. Isolation and partial characterization of a novel amino sugar-containing fucan sulfate from commercial fucus vesiculosus fucoidan. Carbohydr Res. 1994;255:213-224.

45. Sandri G, Bonferoni MC, Rossi S, Ferrari F, Boselli C, Caramella C. Insulin-loaded nanoparticles based on $\mathrm{N}$-trimethyl chitosan: in vitro (Caco-2 model) and ex vivo (excised rat jejunum, duodenum, and ileum) evaluation of penetration enhancement properties. AAPS Pharm Sci Tech. 2010;11(1):362-371.

46. Gaumet M, Vargas A, Gurny R, Delie F. Nanoparticles for drug delivery: the need for precision in reporting particle size parameters. Eur J Pharm Biopharm. 2008;69(1):1-9.

47. Liu ZG, Jiao YP, Liu FN, Zhang ZY. Heparin/chitosan nanoparticle carriers prepared by polyelectrolyte complexation. J Biomed Mater Res A. 2007;83(3):806-812.

48. Vauthier C, Cabane B, Labarre D. How to concentrate nanoparticles and avoid aggregation? Eur J Pharm Biopharm. 2008;69(2):466-475.

49. Iovu M, Dumais G, du Souich P. Anti-inflammatory activity of chondroitin sulfate. Osteoarthritis Cartilage. 2008;16(Suppl 3):S14-S18.

50. Asimakopoulou AP, Theocharis AD, Tzanakakis GN, Karamanos NK. The biological role of chondroitin sulfate in cancer and chondroitin-based anticancer agents. In Vivo. 2008;22(3):385-389.

51. Yang H, Liu S, Cai HW, et al. Chondroitin sulfate as a molecular portal that preferentially mediates the apoptotic killing of tumor cells by penetratin-directed mitochondria-disrupting peptides. J Biol Chem. 2010;285(33):25666-25676.
International Journal of Nanomedicine

\section{Publish your work in this journal}

The International Journal of Nanomedicine is an international, peerreviewed journal focusing on the application of nanotechnology in diagnostics, therapeutics, and drug delivery systems throughout the biomedical field. This journal is indexed on PubMed Central, MedLine, CAS, SciSearch ${ }^{\circledR}$, Current Contents ${ }^{\circledR} /$ Clinical Medicine,

\section{Dovepress}

Journal Citation Reports/Science Edition, EMBase, Scopus and the Elsevier Bibliographic databases. The manuscript management system is completely online and includes a very quick and fair peer-review system, which is all easy to use. Visit http://www.dovepress.com/ testimonials.php to read real quotes from published authors. 